\title{
Prospective evaluation of incobotulinumtoxinA in the management of the masseter using two different injection techniques
}

This article was published in the following Dove Press journal:

Clinical, Cosmetic and Investigational Dermatology

\begin{abstract}
Andreas Nikolis ${ }^{1-4}$
Kaitlyn M Enright ${ }^{1,3-5}$

Sofia Masouri ${ }^{5}$

Steven Bernstein ${ }^{6}$

Christina Antoniou ${ }^{7}$

'Victoria Park Medispa, Westmount, 2Department of Plastic Surgery, University of Montreal, ${ }^{3} \mathrm{VP}$ Research Unit, ${ }^{4}$ Erevna Innovations Inc., ${ }^{5}$ Department of Experimental Surgery, McGill University, Montreal, QC, Canada; 'Department of Dermatology, University of Montreal, Montreal, QC, Canada; ${ }^{7}$ Department of Dermatology, National and Kapodistrian University of Athens, Athens, Greece
\end{abstract}

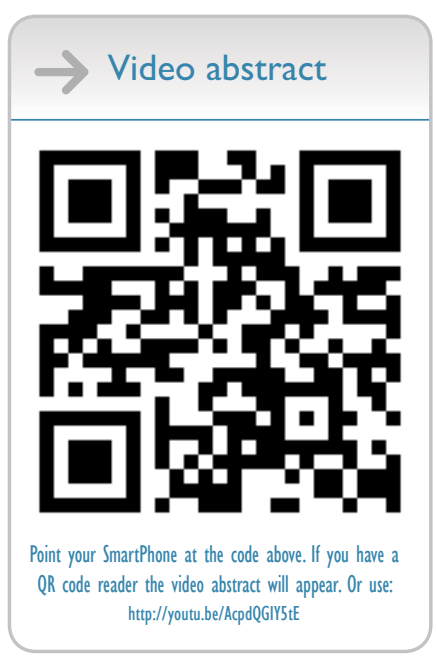

Correspondence: Andreas Nikolis Victoria Park Medispa, 376 Victoria Avenue, \# 400, Westmount, QC H3Z IC3, Canada

$\mathrm{Tel}+$ I 5 I 44887722

Email anikolis@vicpark.com
Background: IncobotulinumtoxinA (Xeomin Cosmetic ${ }^{\circledR}$ ) has been used previously in the management of masseteric hypertrophy. However, a standardized injection technique has not been established. The goal of the present study was to evaluate the efficacy and safety of two injection techniques in the management of masseteric hypertrophy using incobotulinumtoxinA. Methods: Thirty female patients with masseteric hypertrophy were recruited and evenly randomized to receive bilateral treatments of either a single-injection technique (SIT) or a multiinjection technique (MIT). Improvement of masseteric hypertrophy was assessed at week 16 using standardized measurements and photographs. Patients completed a 5-point satisfaction questionnaire while physicians completed the Global Aesthetic Improvement Scale (GAIS) and 10-point photonumeric masseter prominence rating scale.

Results: There were no significant differences in physician ratings on the photonumeric scale and GAIS between the SIT and MIT groups. Results of the standardized measurements also revealed no significant difference between injection techniques. Majority of patients at every visit reported being "satisfied" with treatment results. Clinically, the number and severity of adverse events were similar between groups.

Conclusion: This study supports the noninferiority of both SIT and MIT with regard to efficacy and safety in the management of masseteric hypertrophy, using incobotulinumtoxinA.

Keywords: Xeomin Cosmetic ${ }^{\circledR}$, masseteric hypertrophy, noninferiority, botulinum toxin type A

\section{Background and study rationale}

Masseter muscle hypertrophy is defined by a soft fullness, near the angle of the mandible. A severe enough form of masseter hypertrophy may be cosmetically disfiguring. The first published description of masseter hypertrophy was documented in $1880 .{ }^{1}$ Since that time, $>250$ cases have been reported in published literature. Factors that have been attributed to the etiology of masseter muscle hypertrophy include emotional stress, chronic bruxism, masseteric hyperfunction and parafunction, and microtrauma. ${ }^{2}$ Masseter muscle hypertrophy has also been shown to be a result of compensatory enlargement that functionally compensates for disproportions in fiber-type compositions. Most frequently, masseter muscle hypertrophy occurs among Pacific Asians and is associated with dietary habits and ethnic characteristics. ${ }^{3}$

The appeal of masseteric injections in the female Asian population is that of an oval- and almond-shaped jaw. ${ }^{4}$ The excessive hypertrophic masseters in this population of patients lead to a round face with the lack of any distinctive lower jawline attributes. ${ }^{5}$ This also leads to an appearance of an excessively heavy lower third of the face in such patients. ${ }^{6-8}$ 
The Western jawline in the female involves a delicate balance of a stronger masseteric or jawline in the lower third of the face, which outweighs the creation of a thinned out lower jaw. As such, the lower third of the Western female jaw is one in which the sculpted appearance should remain in place while removing excess masseteric hypertrophy. The goal of which is not to overly thin out the masseters. ${ }^{6}$

Botulinum toxin type A for contouring the lower face is an easy alternative to surgery. ${ }^{9,10}$ Intramuscular injection of botulinum toxin type A inhibits the release of acetylcholine into the neuromuscular junction leading to the temporary partial denervation in the area. ${ }^{11}$ Over a 4-6-week period, the atrophy continues to develop, and there are both a generalized reduction in fiber diameters and fiber size variability. Recovery and reversibility of fiber atrophy occur over 4-6 months. ${ }^{8,12-14}$

IncobotulinumtoxinA (Xeomin Cosmetic ${ }^{\circledR}$; Merz Pharma Canada Ltd., Burlington, ON, Canada) is one of the purest commercially available botulinum toxin type As on the market. It has been approved by Health Canada since 2012 for the temporary improvement in the appearance of moderate-to-severe glabellar lines. ${ }^{15}$ IncobotulinumtoxinA has been shown to be comparable in safety and efficacy to onabotulinumtoxinA in $>3000$ patients studied in clinical trials ranging from Phase I to Phase IV studies at a 1:1 dose conversion ratio. One of the comparative studies, by Lee et al (2014), ${ }^{16}$ was a randomized, double-blind, split-face study where 56 patients were randomized to receive onabotulinumtoxinA (Botox ${ }^{\circledR}$, Vistabel; Allergan Inc., Irvine, CA, USA) or incobotulinumtoxinA (Xeomin ${ }^{\circledR}$, Bocouture; Merz Pharmaceuticals, Frankfurt, Germany) on each side of their face. The results of the study showed that incobotulinumtoxinA was noninferior to onabotulinumtoxinA in terms of efficacy and safety. ${ }^{15}$

Nonetheless, varying injection techniques used in previous studies make comparison difficult and may affect the safety and efficacy of incobotulinumtoxinA in the treatment of masseteric hypertrophy.

As such, there are two particular techniques that require evaluation:

1. A single-injection technique (SIT) involving $40 \mathrm{U}$ of incobotulinumtoxinA delivered directly into the region where the three heads overlap.

2. A standard multiinjection technique (MIT) involving a distribution of $40 \mathrm{U}$ ( $8 \mathrm{U}$ distributed in five different areas) of incobotulinumtoxinA over the width of the masseter while respecting the upper limit, the anterior border of the masseter and the inferior insertion of the masseter. The injections are separated by a $1 \mathrm{~cm}$ distance, and the dose is equally distributed across these sites. The first injection is placed in the center of the muscle, and the remaining injections are placed in an equidistant square format at $1 \mathrm{~cm}$ distances from the center.

Therefore, the goal of this study was to evaluate the efficacy and safety of two injection techniques in the management of masseteric hypertrophy using incobotulinumtoxinA.

\section{Study design}

\section{Ethical approval and informed consent}

This study including its protocol, supplemental documents such as patient consent to photography, the principle investigator, and clinical site received unconditional approval from an independent, central review board (the Quebec Institutional Review Board Services). All patients signed an informed consent document before any study-related procedures were performed. A description of the study procedures is available on ClinicalTrials.gov under the identifier NCT03376464.

\section{Recruitment}

Thirty patients with masseteric hypertrophy were recruited for this study. For each of the 30 patients, there were 60 unique hemiface observations. Inclusion criteria included female gender, $>18$ years, having an established hypertrophy of the masseters, accepting the obligation not to receive any other facial procedures during the 6-month follow-up, understanding and accepting the obligation to appear for all scheduled follow-up visits, no previous facial fillers for 6 months prior to this study, no previous facial fillers along the jawline for 18 months, no previous botulinum toxin type A treatment for masseteric hypertrophy in the last 12 months, and providing informed consent. Exclusion criteria included current pregnancy or lactation, hypersensitivity to incobotulinumtoxinA, generalized disorders of muscle activity (eg, myasthenia gravis and Lambert-Eaton syndrome), presence of infection at the site of injection, inability to comply with follow-up and abstain from facial injections during the study period and having a score of $\geq 2$ on the Merz Aesthetics Scale ${ }^{\mathrm{TM}}$ for Jawline.

\section{Procedures}

Patients were seen during four visits occurring over a period of 6 months. Visits consisted of one treatment of incobotulinumtoxinA for masseter hypertrophy and three follow-ups. 


\section{Visit I (day 0 - baseline)}

Patients signed the informed consent form and, then, underwent screening evaluation. Standardized 3D photography was taken with their face at rest and during jaw clenching. A blinded evaluator completed photonumeric rating scales of masseter prominence (Figure 1) and face shape (Figure 2). Patients were randomized to receive either SIT or MIT and, then, received bilateral treatment with incobotulinumtoxinA as per their randomization schedule. The incobotulinumtoxinA was diluted into $100 \mathrm{U}$ vials in $1 \mathrm{cc}$ of preservative-free saline, and the vial was inverted and swirled three times prior to administration to ensure proper reconstitution. Injections of incobotulinumtoxinA were performed by the investigators. Data on adverse events (AEs) were collected.

\section{Visit 2 (week 6), visit 3 (week 16), and visit 4 (week 20)}

Standardized 3D photography was taken with their face at rest and during jaw clenching. The blinded evaluator completed their photonumeric rating sales and the Global Aesthetic Improvement Scale (GAIS), and patients com-

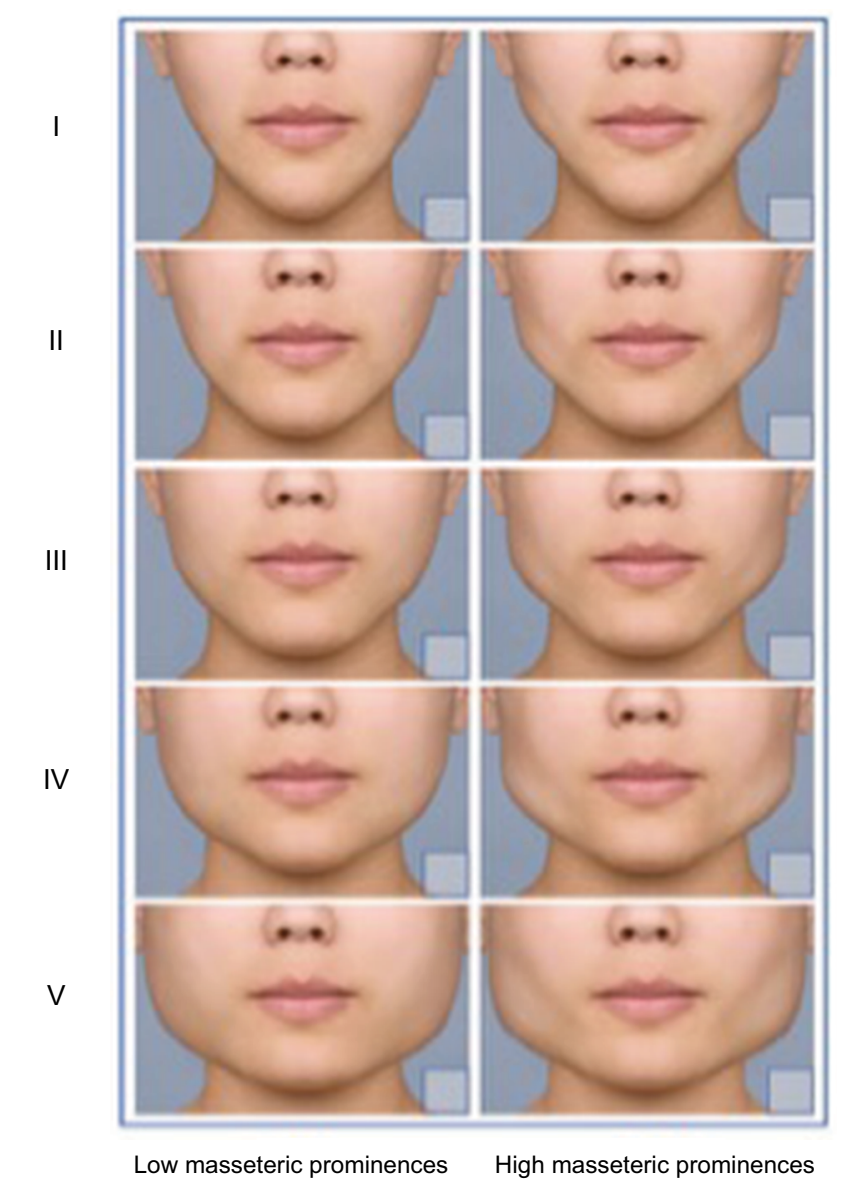

Figure I Ten-point photonumeric masseter prominence rating scale for females. Note: () Merz Pharmaceuticals, GmbH. pleted the Patient Satisfaction Questionnaire. Data on AEs were collected.

\section{Data analyses}

Using an established algorithm, the improvement of masseteric hypertrophy was assessed using two standardized masseter improvement measurements. To calculate these measurements, the midpoint of the intercanthal (ICM) distance was identified. Given that this distance does not vary over time in the adult, this technique provided an anchor point for the remaining measurements. A vertical line was drawn inferior to this and through to the gonion $\left(\mathrm{G}^{\prime}\right)$. The maximal masseteric (MM) protrusion point was identified. A triangle between ICM, $\mathrm{G}^{\prime}$, and MM was drawn (Figure 3). Measurement 1 was the angle between the MM and the ICM vertical line (the ICM angle). This line was measured, and the changes it underwent following treatment were recorded. Measurement 2 was the horizontal distance between the MM and the ICM vertical line. This distance (the MM to midline distance) was measured, and the changes it underwent following treatment were recorded. Data on the ICM angle and MM to midline distance were recorded for patients with their face at rest and while clenching their jaw.

Paired sample $t$-test evaluated whether there was a significant difference between the ICM angles and MM to midline distances between visits on either side. One-way ANOVA was used to reveal if there was a significant difference between the ICM angles and MM to midline distances between the SIT and MIT groups, at each visit, on either side. Significance for all inferences was set to 0.05 . Continuous variables were summarized in tables and include the mean and standard deviation (SD). Categorical variables are presented in tables as frequencies and percentages.

The primary endpoint was the difference in physicianassessed efficacy as per the standardized metric masseter improvement measures between the two different injection techniques at 16 weeks (visit 3 ) in comparison to baseline (visit 1). Secondary endpoints included the reviewer-assessed masseter photonumeric esthetic rating scales between SIT and MIT at 16 weeks in comparison to baseline and the Patient Satisfaction Questionnaire and the GAIS at all visits.

\section{Results}

Thirty women with a mean age of 31.06 years (range 19-51 years; SD 7.17) were enrolled in this study and received bilateral treatment with incobotulinumtoxinA for masseteric hypertrophy, for a total of 60 unique hemiface observations (Figures 4-7). Eighty percent $(n=24)$ of participants were Caucasian, 


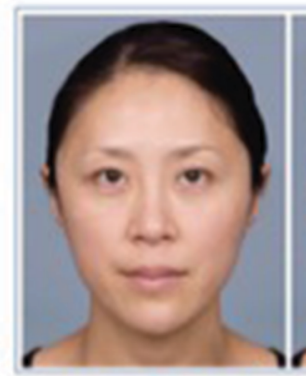

Inverted triangle/ diamond

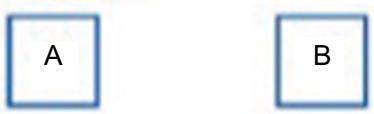

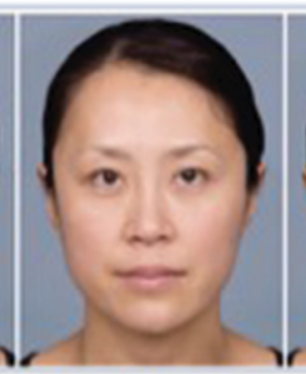

Oblong/Rectangle

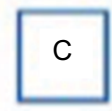

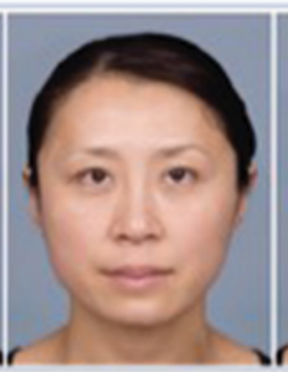

Round

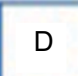

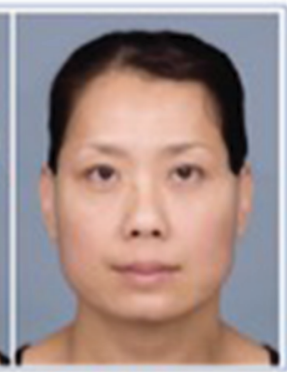

Square/triangle

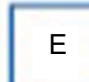

Please indicate your choice by checking the appropriate box

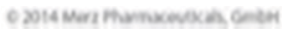

Figure 2 The Merz five-point photonumeric facial shape categories for females. Note: (c) Merz Pharmaceuticals, GmbH.

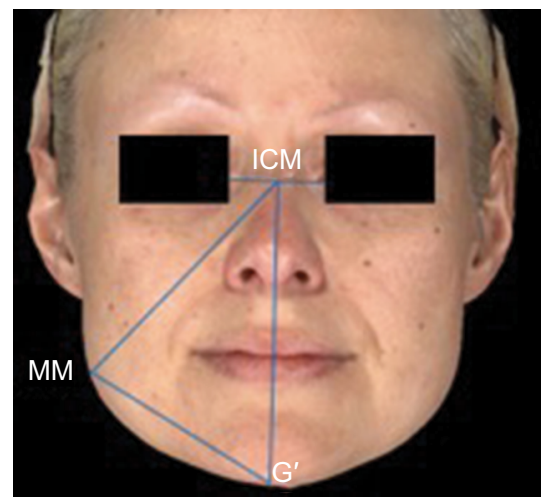

Figure 3 Overview of the standardized masseter improvement measurements. Abbreviations: $\mathrm{G}^{\prime}$, gonion; ICM, midpoint of the intercanthal; MM, maximal masseteric

and $20 \%(\mathrm{n}=6)$ were Asian descent. Fifteen subjects received a SIT, and 15 subjects received the MIT. The mean age of the SIT group was 31.2 years (SD 7.9), and the mean age of the MIT group was 30.9 years (SD 7.17). Four patients were lost to follow-up after visit 1 (treatment), for a drop out rate of $13 \%$.

\section{Primary endpoint}

Metric masseteric hypertrophy improvement outcomes between SIT and MIT at 16 weeks in comparison to baseline

Table 1 displays the mean and SD of the ICM angles and MM to midline distances between all visits for nonclenching and clenching data on the right and left sides of the face. Results of paired sample $t$-tests for the ICM angle and MM to midline distance for nonclenching and clenching data on both the right and left sides of the face are displayed in Table 2. Results of one-way ANOVA comparing the mean and SD of the ICM angles and MM to midline distances between the SIT and MIT groups are displayed in Table 3 for nonclenching data and Table 4 for clenching data.

\section{Nonclenching}

Using paired sample $t$-tests, both the ICM angle and MM to midline distance significantly decreased between baseline and visit 3 (week 16) for nonclenching data on both the right $(P \leq 0.001)$ and left sides of the face $(P \leq 0.001$; Table 2$)$. Oneway ANOVA found that there were no statistically significant differences between the mean of the ICM angles and MM to midline distances between the SIT and the MIT groups, at baseline or visit 3 , for either the right side of the face $(P>0.05)$ or the left side of the face $(P>0.05$; Table 3$)$.

\section{Clenching}

Using paired sample $t$-tests, both the ICM angle and MM to midline distance significantly decreased between baseline and visit 3 (week 16) for clenching data on both the right $(P \leq 0.005)$ and left sides of the face $(P \leq 0.001$; Table 2$)$. Oneway ANOVA found that there were no statistically significant 


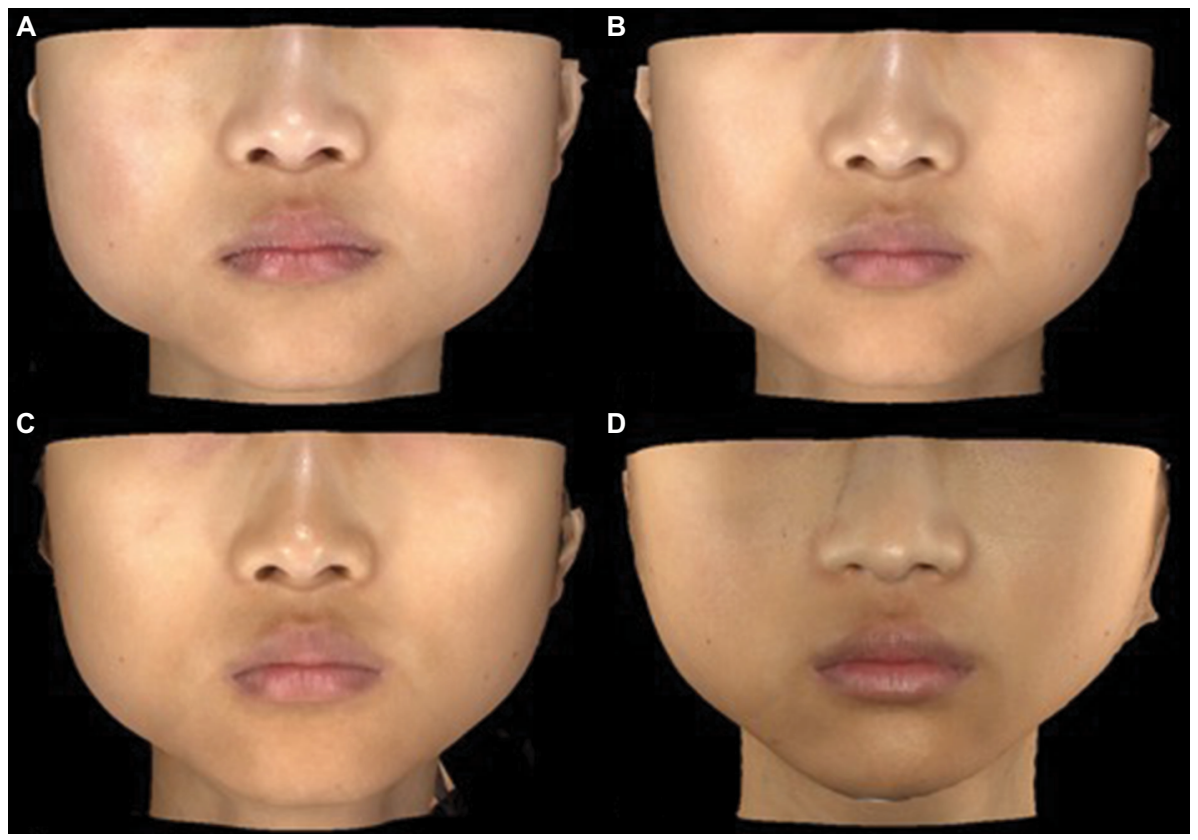

Figure 4 Patient at baseline (A), week 6 (B), week 16 (C), and week 20 (D) after the injection of incobotulinumtoxinA using MIT for the treatment of masseter hypertrophy. Abbreviation: MIT, multiinjection technique.

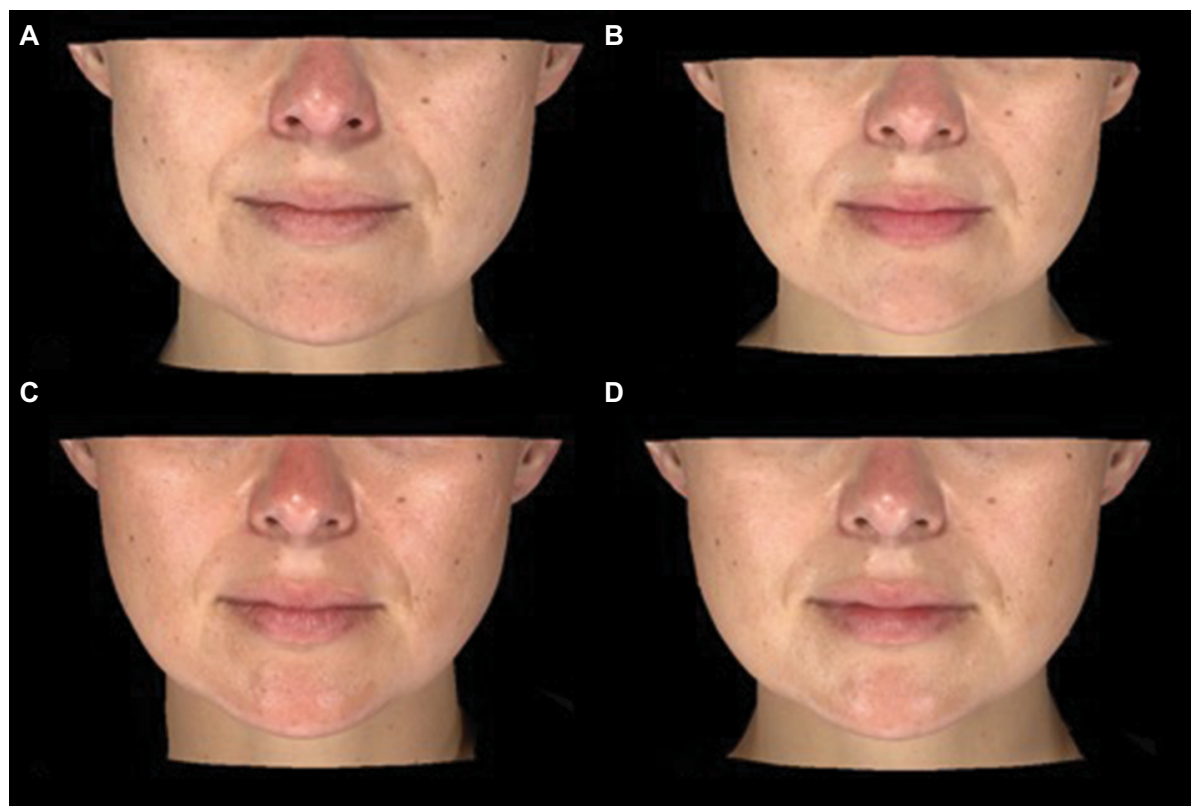

Figure 5 Patient at baseline (A), week 6 (B), week 16 (C), and week 20 (D) after the injection of incobotulinumtoxinA using SIT for the treatment of masseter hypertrophy. Abbreviation: SIT, single-injection technique.

differences between the mean of the ICM angles and MM to midline distances between the SIT and the MIT groups, at baseline or visit 3 , for either the right side of the face $(P>0.05)$ or the left side of the face $(P>0.05$; Table 4$)$.

\section{Secondary endpoints}

Masseter photonumeric esthetic rating scales between SIT and MIT at 16 weeks in comparison to baseline

\section{Face shape}

Fifteen patients did not change face shape categories between visit 1 and visit 3. Eight patients decreased one face shape category (eg, from square to round), two patients decreased two face shape categories (eg, square to oblong), and one patient moved up one face shape category from visit 1 to visit 3 (eg, oblong to round). Face shape category remained consistent between visits $2-4$ for all patients. 


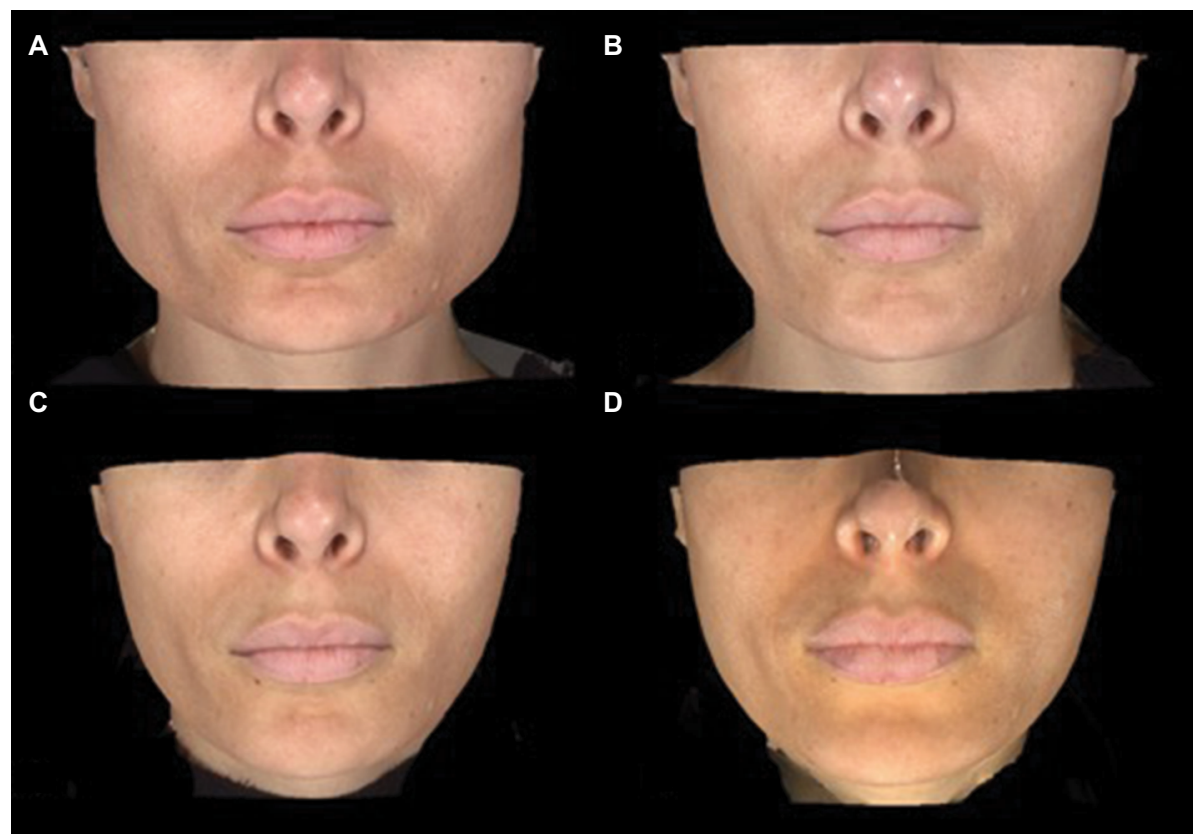

Figure 6 Patient at baseline (A), week 6 (B), week 16 (C), and week 20 (D) after an MIT of incobotulinumtoxinA in the masseters for the treatment of masseter hypertrophy. Abbreviation: MIT, multiinjection technique.

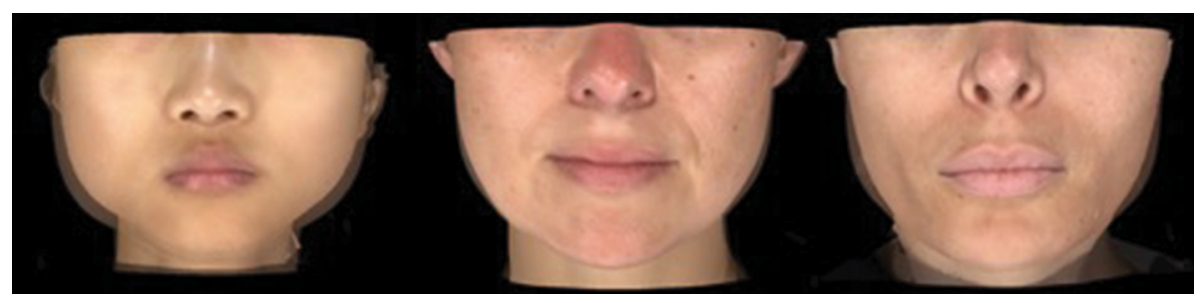

Figure 7 Baseline and visit 3 (week 16) photos superimposed, for three patients treated with incobotulinumtoxinA for masseter hypertrophy.

Table I Mean and SD of the ICM angle and MM to midline distance for nonclenching and clenching data on the right and left sides of the face

\begin{tabular}{|c|c|c|c|c|c|c|c|c|}
\hline \multirow[t]{2}{*}{ Masseter } & \multicolumn{4}{|c|}{ Angle, mm } & \multicolumn{4}{|c|}{ Distance, $\mathbf{m m}$} \\
\hline & Visit I & Visit 2 & Visit 3 & Visit 4 & Visit I & Visit 2 & Visit 3 & Visit 4 \\
\hline \multicolumn{9}{|c|}{ Nonclenching } \\
\hline \multicolumn{9}{|c|}{ Right side } \\
\hline Mean & 43.90 & 42.56 & 42.14 & 42.86 & 76.69 & 70.81 & 73.26 & 74.64 \\
\hline SD & 3.85 & 3.38 & 3.08 & 3.38 & 6.12 & 16.57 & 5.01 & 6.57 \\
\hline \multicolumn{9}{|l|}{ Left side } \\
\hline Mean & 44.67 & 43.18 & 42.20 & 43.07 & 79.19 & 75.23 & 73.49 & 75.15 \\
\hline SD & 3.57 & 3.75 & 3.36 & 3.58 & 4.51 & 5.35 & 4.60 & 5.30 \\
\hline \multicolumn{9}{|l|}{ Clenching } \\
\hline \multicolumn{9}{|l|}{ Right side } \\
\hline Mean & 45.32 & 44.58 & 44.06 & 43.90 & 77.69 & 76.62 & 75.58 & 74.82 \\
\hline SD & 4.28 & 4.14 & 4.19 & 4.30 & 6.51 & 6.81 & 6.42 & 7.16 \\
\hline \multicolumn{9}{|l|}{ Left side } \\
\hline Mean & 47.06 & 45.38 & 45.10 & 44.61 & 81.77 & 76.95 & 77.22 & 75.48 \\
\hline SD & 3.38 & 3.72 & 3.40 & 3.37 & 4.70 & 5.30 & 3.87 & 5.19 \\
\hline
\end{tabular}

Notes: Visit I, baseline; visit 2, week 6; visit 3, week 16; visit 4, week 20. Angle (ICM angle), the angle between the MM and the ICM vertical line. Distance (MM to midline distance), the horizontal distance between the MM and the ICM vertical line.

Abbreviations: ICM, midpoint of the intercanthal; MM, maximal masseteric; SD, standard deviation. 
Table 2 Results of paired samples' $t$-tests for the ICM angle and $M M$ to midline distance for nonclenching and clenching data on the right and left sides of the face

\begin{tabular}{|c|c|c|c|c|}
\hline $\begin{array}{l}\text { Masseter } \\
\text { muscle } \\
\text { positioning } \\
\end{array}$ & Pairs & $\begin{array}{l}\text { Mean } \\
\text { difference, } \\
\text { mm }\end{array}$ & SD & $\begin{array}{l}\text { Significance } \\
\text { (two tailed) }\end{array}$ \\
\hline \multicolumn{5}{|c|}{ Nonclenching } \\
\hline \multirow[t]{6}{*}{ Right side } & Visit I-visit 2 angles & 1.63 & 2.44 & 0.005 \\
\hline & Visit I-visit 3 angles & 1.60 & 1.90 & 0.001 \\
\hline & Visit I-visit 4 angles & 1.39 & 2.28 & 0.023 \\
\hline & Visit I-visit 2 distances & 6.00 & 14.61 & 0.061 \\
\hline & Visit I-visit 3 distances & 3.46 & 2.61 & 0.000 \\
\hline & Visit I-visit 4 distances & 3.36 & 3.27 & 0.001 \\
\hline \multirow[t]{6}{*}{ Left side } & Visit I-visit 2 angles & 1.50 & 2.58 & 0.013 \\
\hline & Visit I-visit 3 angles & 2.23 & 2.50 & 0.000 \\
\hline & Visit I-visit 4 angles & 1.16 & 2.16 & 0.042 \\
\hline & Visit I-visit 2 distances & 4.08 & 3.24 & 0.000 \\
\hline & Visit I-visit 3 distances & 5.72 & 3.56 & 0.000 \\
\hline & Visit I-visit 4 distances & 3.96 & 3.82 & 0.001 \\
\hline \multicolumn{5}{|l|}{ Clenching } \\
\hline \multirow[t]{6}{*}{ Right side } & Visit I-visit 2 angles & 0.99 & 1.29 & 0.002 \\
\hline & Visit I-visit 3 angles & 1.29 & 1.89 & 0.004 \\
\hline & Visit I-visit 4 angles & 1.58 & 1.87 & 0.003 \\
\hline & Visit I-visit 2 distances & 1.49 & 2.36 & 0.007 \\
\hline & Visit I-visit 3 distances & 2.50 & 2.99 & 0.001 \\
\hline & Visit I-visit 4 distances & 3.35 & 4.09 & 0.004 \\
\hline \multirow[t]{6}{*}{ Left side } & Visit I-visit 2 angles & 1.78 & 2.09 & 0.001 \\
\hline & Visit I-visit 3 angles & 1.74 & 2.26 & 0.001 \\
\hline & Visit I-visit 4 angles & 2.11 & 2.51 & 0.003 \\
\hline & Visit I-visit 2 distances & 5.17 & 3.54 & 0.000 \\
\hline & Visit I-visit 3 distances & 4.72 & 3.82 & 0.000 \\
\hline & Visit I-visit 4 distances & 6.42 & 5.37 & 0.000 \\
\hline
\end{tabular}

Notes: Visit I, baseline; visit 2, week 6; visit 3, week 16; visit 4, week 20. Angle (ICM angle), the angle between the MM and the ICM vertical line. Distance (MM to midline distance), the horizontal distance between the MM and the ICM vertical line. Abbreviations: ICM, midpoint of the intercanthal; MM, maximal masseteric.

A one-way ANOVA found no significant differences between the two injection techniques for the physicianassessed face shape $(P=0.888)$ or masseter prominence ratings $(P=0.312)$, at any visit.

A multivariate test for a general linear model revealed no significant effect of injection technique $(P=0.547)$, visit $(P=0.433)$, or injection technique $\times$ visit $(P=0.996)$ on physician-assessed face shape or masseter prominence ratings.

\section{Masseter prominence rating}

Sixteen patients improved in their ratings of masseter prominence. Ten patients remained the same, and none worsened. The proportion of patients who improved was evenly distributed between groups (ie, SIT: $n=8$; MIT: $n=8$ ). The patients who were rated the same between visits were also evenly distributed between groups (ie, SIT: $n=5$; MIT: $n=5$ ).

Sixteen patients did not change in the vertical category of their masseter prominence ratings (ie, categories I-V) between visit 1 and visit 3 . One patient decreased two vertical categories (ie, IV-II), seven patients decreased one vertical category (eg, III-II), and two patients increased one category (ie, III-IV). For the horizontal category of their masseter prominence ratings (ie, low or high), 15 patients remained the same and 11 patients went from having high-to-low masseteric prominences. None of the patients went from having a low prominence to a high one throughout the study.

\section{Patient Satisfaction Questionnaire}

Table 5 displays the results of the Patient Satisfaction Questionnaire at visits $2-4$.

\section{Visit 2}

A total of $31 \%(n=8 / 26)$ of patients were extremely satisfied, $58 \%(n=15 / 26)$ of patients were satisfied, and $11 \%(n=3 / 26)$ of patients were slightly satisfied. No patients were dissatisfied or extremely dissatisfied.

\section{Visit 3}

A total of $15 \%(n=4 / 26)$ of patients were extremely satisfied, $58 \%(n=15 / 26)$ of patients were satisfied, and $27 \%(n=7 / 26)$ of patients were slightly satisfied. No patients were dissatisfied or extremely dissatisfied.

\section{Visit 4}

A total of $4 \%(n=1 / 26)$ of patients were extremely satisfied, $77 \%(n=20 / 26)$ of patients were satisfied, and $19 \%(n=5 / 26)$ of patients were slightly satisfied. No patients were dissatisfied or extremely dissatisfied.

\section{GAIS}

Table 6 displays the results of the physician-evaluated GAIS at visits $2-4$.

\section{Visit 2}

The blinded reviewer found that $12 \%(n=3 / 26)$ of patients very much improved, $65 \%(n=17 / 26)$ of patients much improved, and $23 \%(\mathrm{n}=6 / 26)$ of patients improved since baseline. The reviewer did not report any case where a patient had no change or worsened since baseline.

\section{Visit 3}

The blinded reviewer found that $8 \%(n=2 / 26)$ of patients very much improved, $42 \%(n=11 / 26)$ of patients much improved, and $50 \%(n=13 / 26)$ of patients improved since baseline. The reviewer did not report any case where a patient had no change or worsened since baseline. 
Table 3 Mean and SD of the ICM angle and MM to midline distance of the SIT and MIT groups at all visits for nonclenching data on both sides of the face

\begin{tabular}{|c|c|c|c|c|c|c|c|c|}
\hline \multirow[t]{2}{*}{ Masseter muscle positioning } & \multicolumn{4}{|c|}{ Angle, mm } & \multicolumn{4}{|c|}{ Distance, $\mathbf{m m}$} \\
\hline & Visit I & Visit 2 & Visit 3 & Visit 4 & Visit I & Visit 2 & Visit 3 & Visit 4 \\
\hline \multicolumn{9}{|l|}{ Nonclenching } \\
\hline \multicolumn{9}{|l|}{ Right side } \\
\hline \multicolumn{9}{|l|}{ Mean } \\
\hline SIT & 44.24 & 43.09 & 42.45 & 43.37 & 76.70 & 67.57 & 72.77 & 75.26 \\
\hline MIT & 43.61 & 42.11 & 41.86 & 42.29 & 76.68 & 73.78 & 73.71 & 73.94 \\
\hline \multicolumn{9}{|l|}{ SD } \\
\hline SIT & 4.02 & 3.20 & 3.15 & 3.73 & 6.31 & 23.40 & 5.28 & 7.37 \\
\hline MIT & 3.85 & 3.61 & 3.13 & 3.09 & 6.21 & 5.61 & 4.94 & 5.95 \\
\hline Significance (between groups - ANOVA) & 0.699 & 0.514 & 0.655 & 0.529 & 0.996 & 0.382 & 0.664 & 0.694 \\
\hline \multicolumn{9}{|l|}{ Left side } \\
\hline \multicolumn{9}{|l|}{ Mean } \\
\hline SIT & 44.91 & 42.99 & 42.85 & 43.93 & 79.31 & 74.65 & 74.22 & 76.41 \\
\hline MIT & 44.46 & 43.35 & 41.60 & 42.10 & 79.09 & 75.72 & 72.82 & 73.72 \\
\hline \multicolumn{9}{|l|}{ SD } \\
\hline SIT & 4.17 & 3.05 & 3.87 & 3.83 & 3.99 & 4.62 & 4.79 & 4.94 \\
\hline MIT & 3.15 & 4.37 & 2.85 & 3.25 & 5.06 & 6.05 & 4.51 & 5.65 \\
\hline Significance (between groups - ANOVA) & 0.767 & 0.829 & 0.383 & 0.309 & 0.910 & 0.654 & $0.48 \mathrm{I}$ & 0.311 \\
\hline
\end{tabular}

Notes: Visit I, baseline; visit 2, week 6; visit 3, week 16; visit 4, week 20. Angle (ICM angle), the angle between the MM and the ICM vertical line. Distance (MM to midline distance), the horizontal distance between the MM and the ICM vertical line.

Abbreviations: ICM, midpoint of the intercanthal; MIT, multiinjection technique; MM, maximal masseteric; SD, standard deviation; SIT, single-injection technique; ANOVA, analysis of variance.

Table 4 Mean and SD of the ICM angle and MM to midline distance of the SIT and MIT groups at all visits for clenching data on both sides of the face

\begin{tabular}{|c|c|c|c|c|c|c|c|c|}
\hline \multirow[t]{2}{*}{ Masseter } & \multicolumn{4}{|c|}{ Angle, mm } & \multicolumn{4}{|c|}{ Distance, $\mathbf{m m}$} \\
\hline & Visit I & Visit 2 & Visit 3 & Visit 4 & Visit I & Visit 2 & Visit 3 & Visit 4 \\
\hline \multicolumn{9}{|l|}{ Clenching } \\
\hline \multicolumn{9}{|l|}{ Right side } \\
\hline \multicolumn{9}{|l|}{ Mean } \\
\hline SIT & 46.17 & 45.34 & 44.68 & 44.23 & 78.79 & 78.04 & 76.14 & 76.08 \\
\hline MIT & 44.60 & 43.94 & 43.44 & 43.54 & 76.77 & 75.44 & 75.01 & 73.40 \\
\hline \multicolumn{9}{|l|}{ SD } \\
\hline SIT & 4.94 & 4.73 & 4.93 & 4.53 & 7.96 & 8.81 & 7.54 & 8.29 \\
\hline MIT & 3.68 & 3.66 & 3.43 & 4.30 & 5.13 & 4.66 & 5.39 & 5.87 \\
\hline Significance (between groups - ANOVA) & 0.381 & 0.445 & 0.504 & 0.752 & 0.462 & 0.386 & 0.693 & 0.460 \\
\hline \multicolumn{9}{|l|}{ Left side } \\
\hline \multicolumn{9}{|l|}{ Mean } \\
\hline SIT & 47.08 & 45.98 & 45.35 & 44.62 & 81.75 & 78.31 & 77.78 & 76.94 \\
\hline MIT & 47.04 & 44.89 & 44.88 & 44.59 & 81.79 & 75.82 & 76.70 & 73.83 \\
\hline \multicolumn{9}{|l|}{ SD } \\
\hline SIT & 3.80 & 4.29 & 3.97 & 3.46 & 5.07 & 5.14 & 3.19 & 5.10 \\
\hline MIT & 3.14 & 3.29 & 2.94 & 3.50 & 4.58 & 5.38 & 4.49 & 5.09 \\
\hline Significance (between groups - ANOVA) & 0.978 & 0.507 & 0.749 & 0.988 & 0.985 & 0.284 & 0.515 & 0.229 \\
\hline
\end{tabular}

Notes: Visit I, baseline; visit 2, week 6; visit 3, week 16; visit 4, week 20. Angle (ICM angle), the angle between the MM and the ICM vertical line. Distance (MM to midline distance), the horizontal distance between the MM and the ICM vertical line.

Abbreviations: ICM, midpoint of the intercanthal; MIT, multiinjection technique; MM, maximal masseteric; SD, standard deviation; SIT, single-injection technique.

\section{Visit 4}

The blinded reviewer found that $35 \%(\mathrm{n}=9 / 26)$ of patients much improved and 65\% (n=17/26) improved since baseline. The reviewer did not report any case where a patient very much improved, had no change, or worsened since baseline.

\section{Adverse events}

AEs following treatment were reported. These included the typical and expected AEs associated with an injection technique and were all mild in nature, lasting between a few hours and a maximum of 7 days. Such AEs included bruising, pain, 
Table 5 Results of the Patient Satisfaction Questionnaire at visits 2-4

\begin{tabular}{|c|c|c|c|c|c|c|}
\hline \multirow[t]{2}{*}{ Ratings } & \multicolumn{2}{|c|}{ Visit 2} & \multicolumn{2}{|c|}{ Visit 3} & \multicolumn{2}{|c|}{ Visit 4} \\
\hline & $\mathbf{n}$ & $\%$ & $\mathbf{n}$ & $\%$ & $\mathbf{n}$ & $\%$ \\
\hline Extremely satisfied & 8 & 31 & 4 & 15 & I & 4 \\
\hline Satisfied & 15 & 58 & 15 & 58 & 20 & 77 \\
\hline Slightly satisfied & 3 & 11 & 7 & 27 & 5 & 19 \\
\hline Dissatisfied & 0 & - & 0 & - & 0 & - \\
\hline Extremely dissatisfied & 0 & - & 0 & - & 0 & - \\
\hline Total & 26 & 100 & 26 & 100 & 26 & 100 \\
\hline
\end{tabular}

Note: Visit 2, week 6; visit 3, week 16; visit 4, week 20.

Table 6 Results of the physician-evaluated Global Aesthetic Improvement Scale at visits 2-4

\begin{tabular}{|c|c|c|c|c|c|c|}
\hline \multirow[t]{2}{*}{ Ratings } & \multicolumn{2}{|c|}{ Visit 2} & \multicolumn{2}{|c|}{ Visit 3} & \multicolumn{2}{|c|}{ Visit 4} \\
\hline & $n$ & $\%$ & $n$ & $\%$ & $\mathrm{n}$ & $\%$ \\
\hline Very much improved & 3 & 12 & 2 & 8 & 0 & - \\
\hline Much improved & 17 & 65 & 11 & 42 & 9 & 35 \\
\hline Improved & 6 & 23 & 13 & 50 & 17 & 65 \\
\hline No change & 0 & - & 0 & - & 0 & - \\
\hline Worse & 0 & - & 0 & - & 0 & - \\
\hline Total & 26 & 100 & 26 & 100 & 26 & 100 \\
\hline
\end{tabular}

Note: Visit 2, week 6; visit 3, week 16; visit 4, week 20.

hematoma, edema, and redness. The amount and severity of AEs did not deviate between the SIT and MIT groups during clinical observation.

\section{Discussion}

There were significant improvements in the metric masseteric hypertrophy outcomes between week 16 (visit 3) and baseline. Both the ICM angle and MM to midline distance significantly decreased between baseline and week 16, during clenching and at rest, on both sides of the face, providing evidence that incobotulinumtoxinA was effective at treating masseter hypertrophy in our patient population. These findings were independent of whether a patient was randomized to receive the SIT or MIT. It appears then that providing a single large dose of incobotulinumtoxinA is as effective at treating masseteric hypertrophy as injecting multiple smaller aliquots of product. The blinded reviewer also reported on the GAIS that patients most often very much improved or improved between visits, attesting to the efficacy of incobotulinumtoxinA as a treatment for masseter hypertrophy. The longevity of results in this study was shown to expand into the 20th week postinjection, with all standardized metric masseteric hypertrophy improvement outcomes remaining below baseline at visit 4 . Clinically, the amount and severity of AEs also did not deviate between the SIT and MIT groups.
Together, these findings support the novel indication of incobotulinumtoxin A as a treatment for patients with moderate masseteric hypertrophy and attest to the efficacy and safety of using either a SIT or an MIT of incobotulinumtoxinA to treat this condition. Furthermore, patient's satisfaction in this study was consistently highly rated, with most patients at every visit reporting "satisfied" with treatment results and no "dissatisfied" or "extremely dissatisfied" responses at any visit.

Investigators did not observe a difference in the efficacy between two different injection techniques at week 16 (visit 3) in comparison to baseline, as per the photonumeric masseter esthetic rating scales and GAIS. These findings were in accordance with the results of the standardized metric masseteric hypertrophy improvement outcomes, which, as described earlier, revealed no significant difference between the ability of the SIT and MIT to improve masseter hypertrophy (ie, decrease in ICM angle and MM to midline distance). Therefore, results of the metric and photonumeric masseter improvement measures and physician's and patient's satisfaction scales support that in clinical practice, the chosen injection method could be left to the attending physician's preference, based on the experience and observation.

This study was successful at meeting its primary and secondary endpoints as well as achieving its four a priori objectives: 1) the researchers evaluated the efficacy of improving masseteric hypertrophy using two different injection techniques and found that both SIT and MIT could achieve clinically and statistically similar improvements; 2) the degree of improvement of masseteric hypertrophy following injection of incobotulinumtoxinA was found to be clinically and statistically significant; 3 ) the longevity of results was evaluated and proven to last up to 20 weeks postinjection; and 4) the safety profile of both injection techniques was evaluated and proven to be safe and effective.

A limitation of this study was that the investigators did not administer functional tests before or after treatments to investigate the treatment effect on jaw strength or smile capacity. While no patients in our sample reported any effect on jaw function or smiling abilities, future studies should take into consideration the possibility that paralysis of risorius and zygomaticus muscles could occur if injections were not correctly placed. Future directions include expanding the study period past 6 months in order to further investigate the longevity of results and evaluating the effect and longevity of a lower dose of neuromodulator. 


\section{Conclusion}

This study demonstrated the noninferiority of both SIT and MIT with regard to efficacy and safety in the management of masseteric hypertrophy, using incobotulinumtoxinA.

\section{Acknowledgments}

The study was presented at the Merz Expert Summit (MEXS), Montreal, QC, Canada, November 10-12, 2017. This study was funded by an unrestricted educational grant from Merz Pharma.

\section{Disclosure}

AN is a consultant, speaker, and research collaborator for Merz Pharma, Galderma, and Allergan. The authors report no other conflicts of interest in this work.

\section{References}

1. Kebede B, Megersa S. Idiopathic masseter muscle hypertrophy. Ethiop J Health Sci. 2011;21(3):209-212.

2. Bas B, Ozan B, Muglali M, Celebi N. Treatment of masseteric hypertrophy with botulinum toxin: a report of two cases. Med Oral Patol Oral Cir Bucal. 2010;15(4):649-652.

3. Al-Muharraqi MA, Fedorowicz Z, Al Bareeq J, Al Bareeq R, Nasser M. Botulinum toxin for masseter hypertrophy. Cochrane Database Syst Rev. 2009;1:CD007510.

4. Gaofeng L, Jun T, Bo P, Bosheng Z, Qian Z, Dongping L. Evaluation and selecting indications for the treatment of improving facial morphology by masseteric injection of botulinum toxin type A. J Plast Reconstr Aesthet Surg. 2010;63(12):2026-2031.

5. Lee SH, Wee SH, Kim H-J, et al. Abobotulinum toxin A and onabotulinum toxin A for masseteric hypertrophy: a split-face study in 25 Korean patients. J Dermatolog Treat. 2013;24(2):133-136.
6. Kim NH, Chung JH, Park RH, Park JB. The use of botulinum toxin type A in aesthetic mandibular contouring. Plast Reconstr Surg. 2005;115(3): 919-930.

7. Zhou R, Pan B, Wang C, Wang D. Mandibular rim trilogy with botulinum toxin injection: reduction, projection, and lift. Facial Plast Surg. 2017;33(1):102-108.

8. Wanitphakdeedecha R, Ungaksornpairote C, Kaewkes A, Sathaworawong A, Lektrakul N, Manuskiatti W. The efficacy of two formulations of botulinum toxin type A for masseter reduction: a split-face comparison study. J Dermatolog Treat. 2017;28(5):443-446.

9. Choe SW, Cho WI, Lee CK, Seo SJ. Effects of botulinum toxin type A on contouring of the lower face. Dermatol Surg. 2005;31(5): 502-507.

10. Cha YR, Kim YG, Kim JH, Kim ST. Effect of unilateral injection of botulinum toxin on lower facial asymmetry as evaluated using three-dimensional laser scanning. Dermatol Surg. 2013;39(6): 900-906.

11. Andrade NN, Deshpande GS. Use of botulinum toxin (botox) in the management of masseter muscle hypertrophy: a simplified technique. Plast Reconstr Surg. 2011;128(1):24e-26e.

12. Kim NH, Park RH, Park JB. Botulinum toxin type A for the treatment of hypertrophy of the masseter muscle. Plast Reconstr Surg. 2010;125(6):1693-1705.

13. Arikan OK, Tan FU, Kendi T, Koc C. Use of botulinum toxin type A for the treatment of masseteric muscle hypertrophy. JOtolaryngol. 2006;35(1): $40-43$.

14. Lee H-J, Kim S-J, Lee K-J, Yu H-S, Baik H-S. Repeated injections of botulinum toxin into the masseter muscle induce bony changes in human adults: a longitudinal study. Korean J Orthod. 2017;47(4):222-228.

15. Xeomin Cosmetic Product Monograph. Merz Pharmaceuticals GmbH. 2015. Available from: http://www.merzcanada.com/files/Xeomin $\% 20$ Product\%20Monograph\%20June\%202013.pdf. Accessed September 2015.

16. Lee JH, Park JH, Lee SK, et al. Efficacy and safety of incobotulinum toxin A in periocular rhytides and masseteric hypertrophy: side-byside comparison with onabotulinum toxin A. J Dermatolog Treat. 2014;25(4):326-330.
Clinical, Cosmetic and Investigational Dermatology

\section{Publish your work in this journal}

Clinical, Cosmetic and Investigational Dermatology is an international, peer-reviewed, open access, online journal that focuses on the latest clinical and experimental research in all aspects of skin disease and cosmetic interventions. This journal is included on PubMed. The manuscript management system is completely online

\section{Dovepress}

and includes a very quick and fair peer-review system, which is all easy to use. Visit http://www.dovepress.com/testimonials.php to read real quotes from published authors 